\title{
The Absorption Band Shapes of a Push-Pull Dye Approaching the Cyanine Limit: A Challenging Case for First Principle Calculations
}

\author{
- Supporting Information -
} Amedeo Capobianco*a, Raffaele Borrelli ${ }^{\mathrm{b}}$, Alessandro Landi ${ }^{\mathrm{a}}$, Amalia Velardo ${ }^{\mathrm{a}}$, and
Andrea Peluso

a) Dipartimento di Chimica e Biologia. Università di Salerno, Via G. Paolo II. I-84084 Fisciano (SA), Italy b) Dipartimento di Scienze Agrarie, Forestali e Alimentari. Università di Torino, Largo Paolo Braccini, 2. I-10095 Grugliasco (TO), Italy

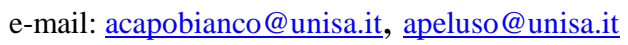

Table S1: Ground and excited state $\left(\mu_{\mathrm{g}}, \mu_{\mathrm{e}}\right.$, Debye) and vertical excitation energies $\left(E_{\mathrm{v}}, \mathrm{eV}\right)$, evaluated by using the $6-31+\mathrm{G}(\mathrm{d}, \mathrm{p})[\mathrm{DZ}]$ and $6-311++\mathrm{G}(2 \mathrm{df}, 2 \mathrm{p})[\mathrm{TZ}]$ basis sets for the gas phase. Excited state properties calculations were computed by using the same basis set employed for ground state geometry optimizations.

\begin{tabular}{|c|c|c|c|c|c|c|}
\hline & \multicolumn{2}{|c|}{$\mu_{\mathrm{g}}$} & \multicolumn{2}{c|}{$\mu_{\mathrm{e}}$} & \multicolumn{2}{c|}{$E_{\mathrm{v}}$} \\
\hline & $\mathrm{DZ} / / \mathrm{DZ}$ & $\mathrm{TZ} / / \mathrm{TZ}$ & $\mathrm{DZ} / / \mathrm{DZ}$ & $\mathrm{TZ} / / \mathrm{TZ}$ & $\mathrm{DZ} / / \mathrm{DZ}$ & $\mathrm{TZ//TZ}$ \\
\hline PBE & 14.6 & 14.4 & 15.0 & 14.7 & 2.36 & 2.36 \\
\hline B3LYP & 14.4 & 14.2 & 15.5 & 15.4 & 2.53 & 2.54 \\
\hline CAM-B3LYP & 13.3 & 13.2 & 16.3 & 16.0 & 2.73 & 2.74 \\
\hline M06-2X & 12.7 & 12.8 & 16.0 & 15.9 & 2.70 & 2.71 \\
\hline$\omega \mathrm{B} 97 \mathrm{X}$ & 12.4 & 12.3 & 16.4 & 16.2 & 2.88 & 2.89 \\
\hline
\end{tabular}

Table S2: Ground and excited state $\left(\mu_{\mathrm{g}}, \mu_{\mathrm{e}}\right.$, Debye) and vertical excitation energies $\left(E_{\mathrm{v}}, \mathrm{eV}\right)$, evaluated by using the $6-31+\mathrm{G}(\mathrm{d}, \mathrm{p})$ [DZ] and $6-311++\mathrm{G}(2 \mathrm{df}, 2 \mathrm{p})$ [TZ] basis sets for 1,4-dioxane. Excited state properties calculations were computed by using the same basis set employed for ground state geometry optimizations.

\begin{tabular}{|c|c|c|c|c|c|c|}
\hline & \multicolumn{2}{|c|}{$\mu_{\mathrm{g}}$} & \multicolumn{2}{c|}{$\mu_{\mathrm{e}}$} & \multicolumn{2}{c|}{$E_{\mathrm{v}}$} \\
\hline & $\mathrm{DZ} / / \mathrm{DZ}$ & $\mathrm{TZ//TZ}$ & $\mathrm{DZ} / / \mathrm{DZ}$ & $\mathrm{TZ} / / \mathrm{TZ}$ & $\mathrm{DZ} / / \mathrm{DZ}$ & $\mathrm{TZ} / / \mathrm{TZ}$ \\
\hline PBE & 18.5 & 18.2 & 18.6 & 18.4 & 2.17 & 2.18 \\
\hline B3LYP & 18.2 & 18.0 & 19.1 & 18.9 & 2.31 & 2.33 \\
\hline CAM-B3LYP & 16.9 & 16.7 & 19.9 & 19.7 & 2.45 & 2.47 \\
\hline M06-2X & 16.2 & 16.2 & 19.6 & 19.5 & 2.42 & 2.44 \\
\hline$\omega B 97 X$ & 15.5 & 15.4 & 20.3 & 20.1 & 2.56 & 2.58 \\
\hline
\end{tabular}


Table S3: Ground and excited state $\left(\mu_{\mathrm{g}}, \mu_{\mathrm{e}}\right.$, Debye) and vertical excitation energies $\left(E_{\mathrm{v}}, \mathrm{eV}\right)$, evaluated by using the $6-31+\mathrm{G}(\mathrm{d}, \mathrm{p})[\mathrm{DZ}]$ and $6-311++\mathrm{G}(2 \mathrm{df}, 2 \mathrm{p})$ [TZ] basis sets for acetonitrile. Excited state properties calculations were computed by using the same basis set employed for ground state geometry optimizations.

\begin{tabular}{|c|c|c|c|c|c|c|}
\hline & \multicolumn{2}{|c|}{$\mu_{\mathrm{g}}$} & \multicolumn{2}{c|}{$\mu_{\mathrm{e}}$} & \multicolumn{2}{c|}{$E_{\mathrm{v}}$} \\
\hline & $\mathrm{DZ} / / \mathrm{DZ}$ & $\mathrm{TZ} / / \mathrm{TZ}$ & $\mathrm{DZ} / / \mathrm{DZ}$ & $\mathrm{TZ} / / \mathrm{TZ}$ & $\mathrm{DZ} / / \mathrm{DZ}$ & $\mathrm{TZ//TZ}$ \\
\hline PBE & 24.4 & 24.1 & 23.6 & 23.4 & 2.20 & 2.20 \\
\hline B3LYP & 24.4 & 24.2 & 23.9 & 23.6 & 2.32 & 2.33 \\
\hline CAM-B3LYP & 23.9 & 23.6 & 24.1 & 23.9 & 2.38 & 2.39 \\
\hline M06-2X & 22.7 & 22.7 & 23.7 & 23.5 & 2.34 & 2.35 \\
\hline$\omega B 97 X$ & 22.9 & 22.6 & 24.5 & 24.3 & 2.38 & 2.40 \\
\hline
\end{tabular}

Figure S1: Predicted UV/absorption spectra for 1/s-trans conformer in dioxane at $298 \mathrm{~K}$.

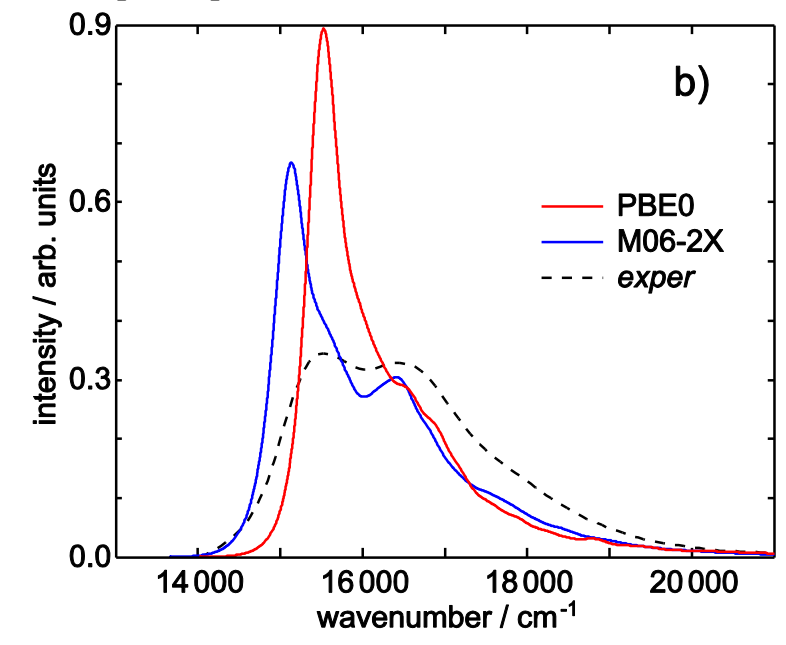

Figure S2: Predicted UV/absorption spectra ( $\omega$ B97X, gas phase, 1/s-trans conformer, $298 \mathrm{~K})$ by using different scaling factors $(s f)$.

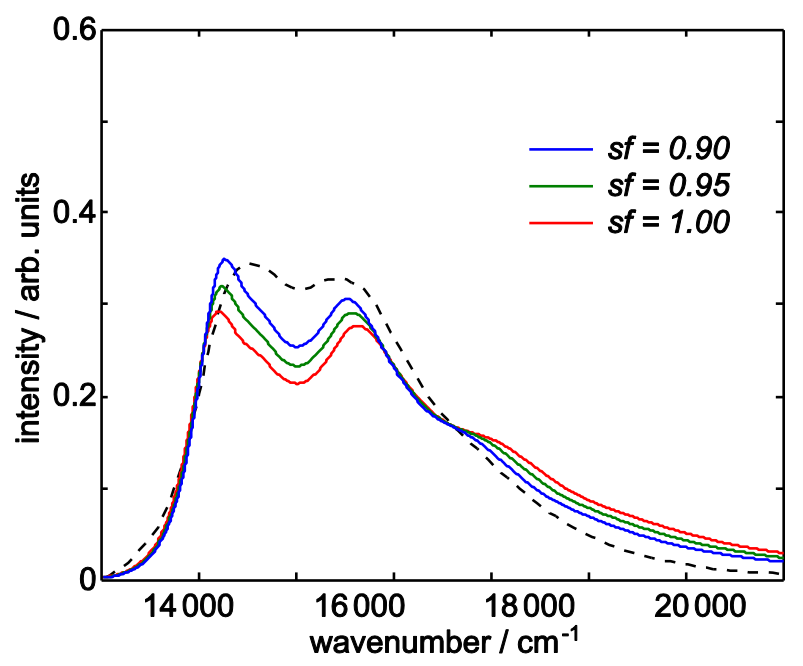


Figure S3: Predicted UV/absorption spectra for the s-trans and s-cis conformers of $\mathbf{1}$ in 1,4-dioxane at 298 K. Panel a), CAM*. Panel b), $\omega$ B97X.
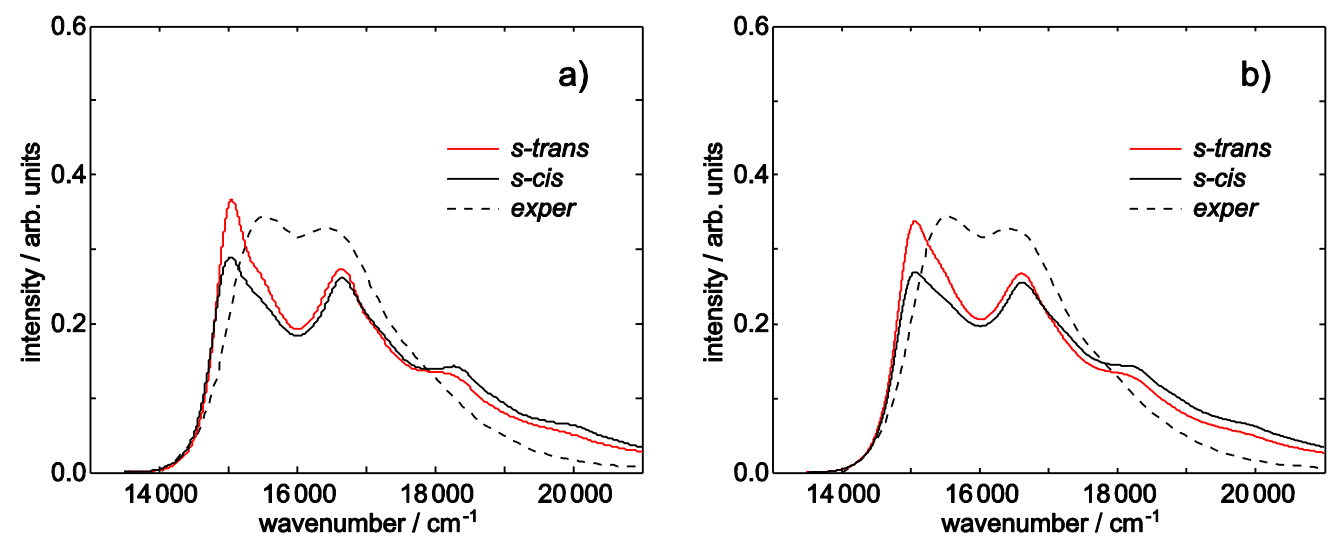

Figure S4: Predicted UV/absorption spectra at $298 \mathrm{~K}$ for $1 / s$-trans conformer, gas phase.
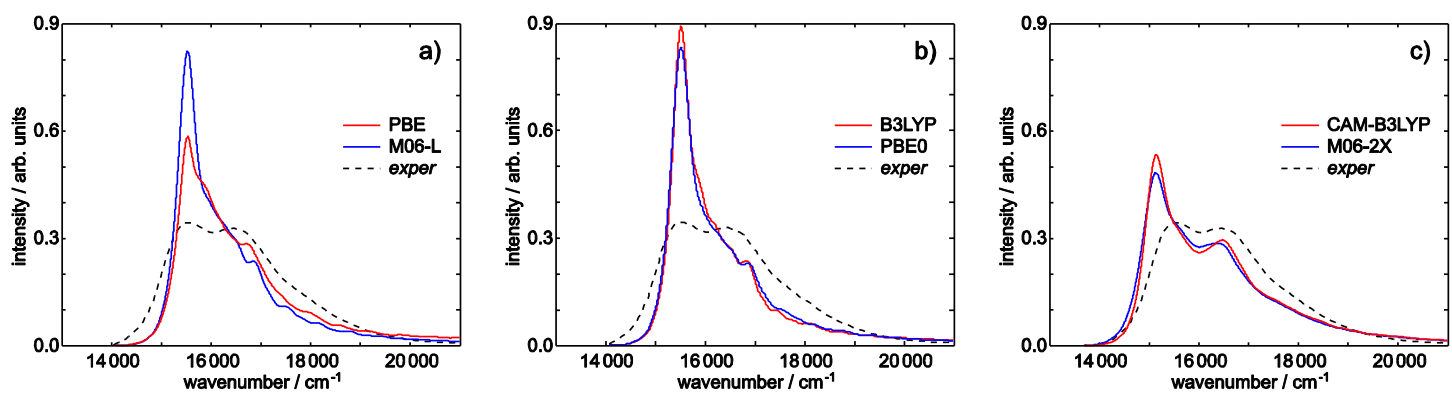

Figure S5: Isosurface contour plots of the HOMO (left) and LUMO (right) of 1, s-trans conformer.
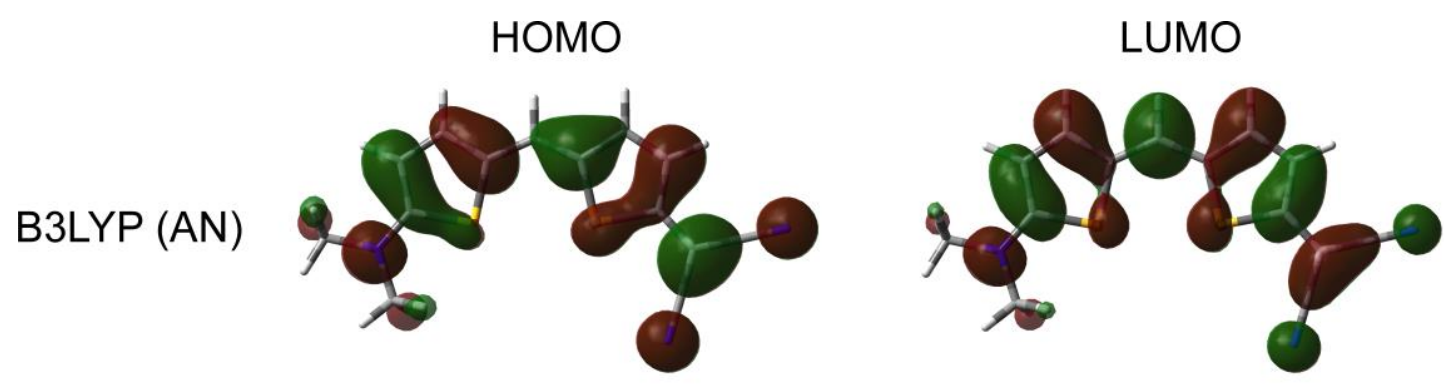

CAM* (gas)
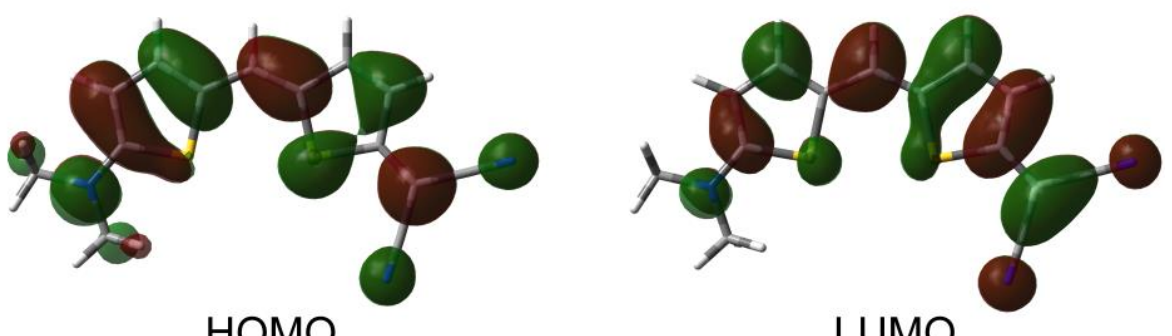

HOMO

LUMO 